\title{
Extensive training, partial reinforcement, and temporal gaps do not affect S-S learning in second-order autoshaping
}

\author{
RUTH M. COLWILL and ROBERT A. RESCORLA \\ University of Pennsylvania, Philadelphia, Pennsylvania
}

\begin{abstract}
A second-order autoshaping procedure was used to examine the effects of three variables on the amount of information that could be learned about the stimulus properties of a reinforcer. All three experiments paired several keylight S2s with different keylight S1s and then carried out discriminative autoshaping with those S1s. Learning about the stimulus properties of S1 was inferred from changes in the response to its paired $S 2$ when that $S 1$ was changed in value. The sensitivity of S2 to changes in S1 was investigated as a function of number of S2-S1 pairings (Experiment 1), partial reinforcement (Experiment 2), and temporal distance between S2 and S1 (Experiment 3). Each experiment found evidence of a selective change in responding to an S2 as a function of the treatment of its S1. However, the amount of that change was not affected by any of the three variables studied. Those results imply that, within the ranges used here, none of these variables changes the degree of learning about the stimulus properties of a reinforcer.
\end{abstract}

Recent studies suggest that a considerable amount is learned about the sensory aspects of the reinforcer in simple Pavlovian conditioning. Much of that evidence comes from experiments in which previously established responding to the conditioned stimulus (CS) is examined after postconditioning changes in the value of the reinforcer. For instance, in a second-order autoshaping procedure in which one keylight, stimulus 1 (S1), is paired with food and then used to establish pecking to another keylight (S2), subsequent changes in the reinforcement of $S 1$ produce substantial changes in responding to $S 2$ (see, e.g., Rashotte, Griffin, \& Sisk, 1977; Rescorla, 1979,1980 ). Because the change is specific to the S2 paired with that $S 1$, one may infer the presence of stimulus-stimulus (S-S) learning.

However, these manipulations only partially modify responding to $\mathbf{S 2}$. For instance, an $\$ 2$ frequently continues to elicit some responding despite elimination of responding to its S1. Moreover, in other preparations (e.g., Holland \& Rescorla, 1975; Rizley \& Rescorla, 1972) and in variations on the autoshaping preparation (e.g., Nairne \& Rescorla, 1981), responding to S2 seems to be unaffected by manipulation of the value of S1. Those observations suggest that some of the responding to $\$ 2$ does not depend on its association with the sensory aspects of the reinforcer. Instead, there may be an association between $\$ 2$ and some aspect of the response pattern evoked in its presence by $S 1$; that is to say, there may be some stimulus-response (S-R) learning.

This research was supported by grants from the National Science Foundation to the University of Pennsylvania. Requests for reprints may be sent to either author at: Department of Psychology, University of Pennsylvania, 3815 Walnut Street, Philadelphia, PA 19104.
Rescorla (1980) has argued that the S-S portion of this learning can be enhanced under some circumstances. For instance, if S2 and S1 are similar or if they occur simultaneously, S-S learning may be encouraged. By contrast, if $S 2$ and $S 1$ are dissimilar or occur sequentially, $S-R$ learning may be magnified. The present paper investigated three other conditions which might be expected to encourage S-R learning: degree of training, percentage of reinforcement, and temporal distance from the reinforcer. These variables were selected because each has been suggested as contributing to the insensitivity of instrumental responding to postconditioning changes in the value of the reinforcer (e.g., Adams \& Dickinson, 1981a; Chen \& Amsel, 1980; Haas, Shessel, Willner, \& Rescorla, 1970; Morgan, 1974, 1979; Rescorla, 1977). If those same variables were identified as encouraging response encoding of the reinforcer in Pavlovian conditioning, the substantial insensitivity to reinforcer changes sometimes seen in instrumental learning might be placed in a broader context.

Experiment 1 examined whether increasing the number of S2-S1 pairings beyond the point of asymptotic conditioning would encourage S-R learning. Several authors (e.g., Adams \& Dickinson, 1981b; Gilhousen, 1933; Irwin, 1971; Kimble \& Perlmuter, 1970; Tolman, 1933, 1948) have suggested that, with extensive pairings of the instrumental response with the reinforcer, the response becomes autonomous in the sense of becoming independent of the current value of its reinforcer. Experiment 2 examined whether pairing $S 2$ and $S 1$ on a partial reinforcement schedule would make $S 2$ independent of the value of $\mathrm{S} 1$. In instrumental learning, partial reinforcement commonly increases resistance to extinction and has been suggested as a technique for increasing resistance 
to changes in the reinforcer value (e.g., Capaldi \& Myers, 1978; Chen \& Amsel, 1980). Experiment 3 investigated the differential sensitivity of several S2s, each presented in sequence prior to an S1, to subsequent changes in the value of that S1. Some authors (e.g., Rescorla, 1977; Wilson, Sherman, \& Holman, 1981) have argued that instrumental behavior often involves sequences of responses that differ in their temporal distance from the reinforcer. They have suggested that perhaps earlier responses in those sequences are reinforced primarily by later responses and their associated stimuli; consequently, those early responses may be less sensitive to changes in the value of the ultimate goal.

All three experiments used a second-order autoshaping procedure with pigeons and assessed S-R learning in terms of the preservation of responding to $S 2$ when $S 1$ was subjected to extinction.

\section{EXPERIMENT 1}

The intention of this experiment was to examine the influence of the number of pairings of S2 with S1 on the continued performance to $\mathrm{S} 2$ when $\mathrm{S} 1$ was changed in value. Pigeons were initially given first-order conditioning with two keylights, blue (B) and an X. Then, B and $X$ were each used to second-order condition two other keylights. One of the two keylights reinforced by B (and one of the two reinforced by $\mathrm{X}$ ) was presented four times more often than the other. This resulted in each S1 conducting extensive training on one $S 2$ and moderate training on another. Then one S1 was extinguished and one S1 received further reinforcement. Finally, responding

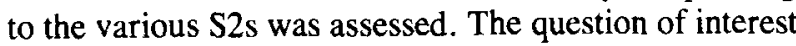
was whether responding to an extensively trained S2 would be more resistant to these manipulations of the value of $\mathrm{S} 1$ than responding to a moderately trained S2 would be.

\section{Method}

Subjects. The subjects were 16 female Carneaux pigeons about 1 year old. They were maintained at $75 \%$ of their free-feeding weights. They had previously participated in an extinction-ofautoshaping experiment. Treatments in the present experiment were arranged to be orthogonal to those experimental histories.

Apparatus. The apparatus consisted of eight identical operant chambers, each measuring $27 \times 27 \times 35 \mathrm{~cm}$. The metal front panel of each chamber had a $5 \times 5 \mathrm{~cm}$ food magazine at its center, located $5 \mathrm{~cm}$ above the wire-mesh floor. There were three response keys, $2.5 \mathrm{~cm}$ in diameter; one was located directly above the hopper and one was located on each side of the center front wall, $20 \mathrm{~cm}$ above the floor. Located behind the left-hand key was an IEE inline projector which permitted the transillumination of the key with color and orientation stimuli. The left-hand half of that key could be illuminated with red $(R)$ or green $(G)$ light, and the right-hand half with a grid of $1-\mathrm{mm}$ black lines spaced $1 \mathrm{~mm}$ apart in a horizontal $(\mathrm{H})$ or vertical (V) orientation on a white background. When either half of the key was illuminated, the other half remained black. In addition, the entire key could be illuminated by a uniform blue (B) stimulus or an X composed of 1-mm black lines on a white background. These stimuli were generated by Ektachrome slides of drawings composed of "Color-aid" artists' paper. The remaining walls and ceiling of the chambers were composed of clear Plexiglas.
These chambers were placed in sound- and light-attenuating shells with ventilation fans providing background noise of $62 \mathrm{~dB}$ (re 20 , $\mu \mathrm{N} / \mathrm{m}^{2}$ ). On the rear wall of those shells was mounted a $6-\mathrm{W}$ bulb that was continuously illuminated during the session except during the operation of the food hopper. That hopper contained Purina Pigeon Grain. Experimental events were controlled and recorded automatically by relays and a microprocessor located in an adjoining room.

Procedure. Because these birds had previously received autoshaping in another experiment, no magazine training was necessary. All birds were given first-order conditioning designed to produce autoshaped responding to $\mathrm{X}$ and $\mathrm{B}$ while yielding low rates of responding to $R, G, H$, and $V$. On each of 4 days, they received 305 -sec presentations of $B$ and $X$, all terminating with $5 \mathrm{sec}$ of food availability. On the next 6 days, they received 3 reinforced and 15 nonreinforced presentations of each of those stimuli. This partial reinforcement of $\mathrm{B}$ and $\mathrm{X}$ was designed to match the frequencies of reinforcement they would be given during their subsequent use as reinforcers in the second-order phase of the experiment. The intertrial interval (ITI) was $60 \mathrm{sec}$ throughout these first-order conditioning sessions. Finally, all subjects received four sessions during each of which there were 3 nonreinforced presentations each of $R, G, H$, and $V$, and 3 reinforced and 15 nonreinforced presentations each of $X$ and $B$. The ITI was lengthened to $75 \mathrm{sec}$.

On each of the next 2 days, $B$ and $X$ were used as reinforcers to second-order condition $\mathrm{R}, \mathrm{G}, \mathrm{H}$, and $\mathrm{V} ; \mathrm{X}$ was used to reinforce the orientations, and $B$ was used to reinforce the colors. Half the subjects received 3 presentations of $H-X$ and $R-B$ and 12 presentations of $V-X$ and $G-B$; the remaining subjects were given 3 presentations of V-X and G-B and 12 presentations of $H-X$ and $R-B$. In addition, all subjects received 3 reinforced presentations each of $\mathrm{X}$ and $\mathrm{B}$. During this phase, the ITI was $75 \mathrm{sec}$ and all stimuli were 5 sec long.

Over the next 10 days, all animals received a treatment designed to extinguish one of the first-order stimuli but to continue reinforcement of the other. Half of the animals received, on each day, 6 reinforced presentations of $B$ and 24 nonreinforced $X$ presentations. The other half of the animals received the same treatment with $B$ and $X$ interchanged. The ITI was $60 \mathrm{sec}$ during this phase.

Finally, two test sessions were administered. The first half of each contained continued first-order discrimination for half the number of trials of a standard discrimination session ( 3 reinforced and 12 nonreinforced presentations); the second half contained 3 nonreinforced presentations each of $R, G, H$, and $V$. In the first half of each session, the ITI was $60 \mathrm{sec}$; in the second half, it was $75 \mathrm{sec}$.

\section{Results and Discussion}

First-order conditioning of $X$ and $B$ proceeded rapidly, as did the discrimination of $R, G, H$, and $V$. However, one bird showed persistent pretest responding to one of the intended second-order stimuli and was consequently dropped from the experiment. By the last day preceding second-order conditioning, the mean responses per minute for $X$ and $B$ were 79 and 87 , whereas those for $R, G$, $H$, and $V$ were $1,11,3$ and 1 , respectively.

Second-order conditioning was also rapid: in the second session, the rate of responding to the second-order stimuli given extensive training was 36 responses/min, while the stimuli receiving only moderate training were responded to at a rate of 38 responses $/ \mathrm{min}$. Somewhat surprisingly, the levels of responding were not higher after many reinforced trials than after a few reinforced trials. Throughout this phase of second-order conditioning, responding to $B$ and $X$ remained high and similar on the various trial types. 


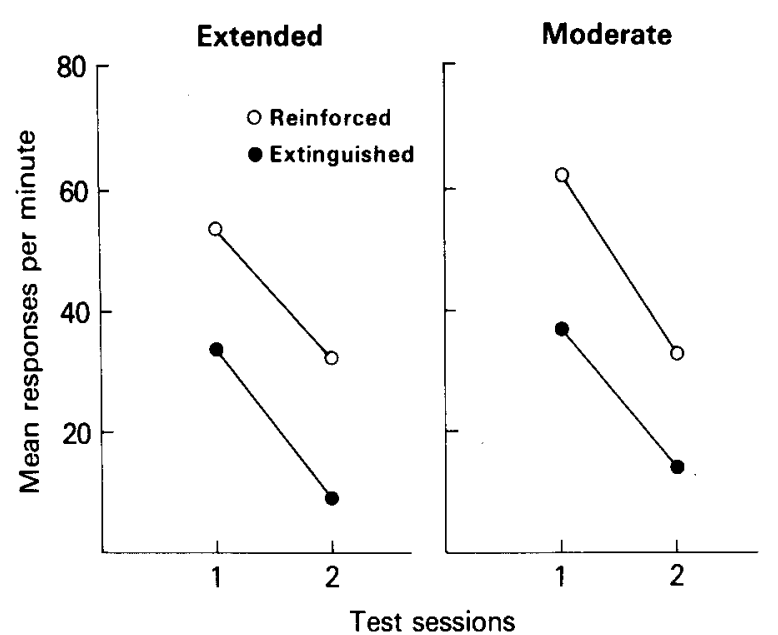

Figure 1. Experiment 1: Second-order responding after the reinforcer had been extinguished (solid symbols) or maintained (open symbols) following extended (left panel) and moderate (right panel) training.

The subsequent discrimination between $\mathrm{B}$ and $\mathrm{X}$ developed somewhat slowly. However, by the final day of this phase, the mean responses per minute for the reinforced and nonreinforced first-order stimuli were 96 and 15 , respectively.

The results of most interest, from the final test sessions with $\mathrm{R}, \mathrm{G}, \mathrm{H}$, and $\mathrm{V}$, are shown in Figure 1. These results are separated according to whether second-order conditioning had been extensive (left panel) or moderate (right panel). Within each panel, responding to the second-order stimuli is separated according to whether the first-order stimulus used was subsequently reinforced or extinguished. Whether training had been moderate or extended, differential treatment of the S1s produced differential responding to the associated S2s. Statistical analysis over both test sessions found reliably less responding to an $S 2$ whose $S 1$ had been extinguished rather than reinforced for both moderate $(T=5, p<.01)$ and extensive $(T=13.5, p<.05)$ training conditions. That effect was of similar magnitude in the two training conditions. For six pigeons, the difference between the extensively trained S2s was greater than the difference between the moderately trained S2s after the differential treatment of the S1s; for seven pigeons, the difference between the moderately trained S2s was greater than that between the extensively trained S2s; for the two remaining subjects, these differences were of the same size.

The differential responding observed here to the various S2s is important, because it demonstrates that, despite the complexity of the task, subjects did learn the individual associations. The character of that learning, however, does not appear to differ as a function of the number of conditioning trials. There is no evidence that the amount of extended training used in the present experiment better preserved responding to $\mathrm{S} 2$ when $\mathrm{S} 1$ was extinguished. It therefore provides no support for the proposition that extended training enhances S-R learning.

\section{EXPERIMENT 2}

The intention of the second study was to examine whether partial reinforcement of S2 by $S 1$ would make $\mathrm{S} 2$ less sensitive to a change in the conditioned status of S1. The design was modeled after that of Experiment 1. Two keylights (B and X) that had been paired with food were each used to second-order condition two other (S2) keylights. For one of the S2s reinforced by B and one reinforced by $\mathrm{X}$, continuous reinforcement was used; for the other S2s, the appropriate reinforcer was presented on only half of its trials. Then, a discrimination between $\mathrm{B}$ and $\mathrm{X}$ was taught and responding to the various S2s was assessed. The question of interest is whether differential treatment of the S1s would have less of an effect on the partially reinforced $S 2$ s than on the continuously reinforced S2s.

\section{Method}

Subjects and Apparatus. The subjects were 16 naive female Carneaux pigeons about 1 year old, maintained at $75 \%$ of their freefeeding weights. The apparatus was that of Experiment 1.

Procedure. The animals were initially trained to eat food from the magazine. On the first day, they were placed in the chambers and the food hoppers were activated until the birds had eaten for about $15 \mathrm{sec}$. Then the hopper presentations were progressively shortened and given less frequently until the birds ate consistently during a 5-sec presentation. On each of the next 3 days, the animals were given 44 hopper deliveries spaced at a mean ITI of $1 \mathrm{~min}$.

All birds were then given first-order conditioning designed to produce autoshaped responding to $X$ and $B$ while yielding low rates of responding to $R, G, H$, and $V$. On each of 4 days, they received 305 -sec presentations of $B$ and $X$, all terminating with $5 \mathrm{sec}$ of food availability. On the next 10 days, they received 3 reinforced and 9 nonreinforced presentations of each of those stimuli. This partial reinforcement of $B$ and $X$ was designed to match the frequencies of reinforcement they would be given during their subsequent use as reinforcers in the second-order phase of the experiment. The ITI was $60 \mathrm{sec}$ throughout these first-order conditioning sessions. Finally, all subjects received four sessions during each of which there were 3 nonreinforced presentations each of $R, G$, $H$, and $V$ and 3 reinforced and 9 nonreinforced presentations each of $X$ and $B$. The ITI was lengthened to 120 sec.

On each of the next 2 days, $B$ and $X$ were used as reinforcers to second-order condition $\mathrm{R}, \mathrm{G}, \mathrm{H}$, and $\mathrm{V} ; \mathrm{X}$ was used to reinforce the orientations, and $B$ was used to reinforce the colors. Half the subjects received six trials each of $H-X$ and $R-B$, three trials each of $\mathrm{V}-\mathrm{X}$ and $\mathrm{G}-\mathrm{B}$, and three nonreinforced presentations of $\mathrm{V}$ and $\mathrm{G}$; the remaining subjects were given six trials each of V-X and G-B, three trials each of $\mathrm{H}-\mathrm{X}$ and $\mathrm{R}-\mathrm{B}$, and three nonreinforced presentations of $H$ and $R$. In addition, all subjects received three reinforced presentations each of X and B. During this phase, the ITI was $120 \mathrm{sec}$ and all stimulus presentations were $5 \mathrm{sec}$.

Over the next 8 days, all animals received a treatment designed to extinguish responding to one of the first-order stimuli but maintain responding to the other. Half of the animals received on each day 6 reinforced presentations of $B$ and 24 nonreinforced $X$ presentations. The other half of the animals received the same treatment with $B$ and $X$ interchanged. The ITI was $60 \mathrm{sec}$ during this phase.

Finally, two test sessions were administered. The first half of each of these sessions continued the discrimination treatments of the first-order stimuli for half the number of trials of a standard discrimination session; the second half of each session contained three nonreinforced presentations each of $R, G, H$, and $V$. In the 
first half of each session, the ITI was $60 \mathrm{sec}$ and in the second half, it was $120 \mathrm{sec}$.

\section{Results and Discussion}

First-order conditioning of $\mathrm{X}$ and $\mathrm{B}$ proceeded rapidly, as did the discrimination of $\mathrm{R}, \mathrm{G}, \mathrm{H}$, and $\mathrm{V}$. By the last day preceding second-order conditioning, the mean responses per minute for $X$ and $B$ were 89 and 100 , whereas those for $\mathrm{R}, \mathrm{G}, \mathrm{H}$, and $\mathrm{V}$ were $3,14,2$, and 1, respectively.

Second-order conditioning of the color and orientation stimuli was very rapid. Responding to the stimuli given continuous reinforcement was greater than responding to the partially reinforced stimuli $(66.7$ and 50.7 responses $/ \mathrm{min}$, respectively) in the final session of training $(\mathrm{T}=15, \mathrm{p}<.02)$. That difference replicates the pattern reported by Rashotte (1981). More important, it suggests that the subjects were sensitive to the differences in the relationship of the various second-order stimuli to reinforcement. Responding to $B$ and $X$ remained high and similar on the various trial types.

Differential responding developed between $\mathrm{B}$ and $\mathrm{X}$ during discrimination training. By the final day of that phase, the mean responses per minute for the reinforced and nonreinforced first-order stimuli were 99 and 16 , respectively.

The results of most interest, from the final test sessions with $R, G, H$, and $V$, are shown in Figure 2. Responding to the continuously reinforced second-order stimuli is shown in the left panel, and that to the partially reinforced stimuli is shown in the right panel. The data for each test session are plotted separately and according to whether the first-order reinforcer had been extinguished or reinforced. First, the graphic data suggest that the differences in terminal acquisition rates of second-order conditioning carried over to the test period. However, statistical analysis did not reveal responding to be significantly higher to the S2s that had been continuously reinforced

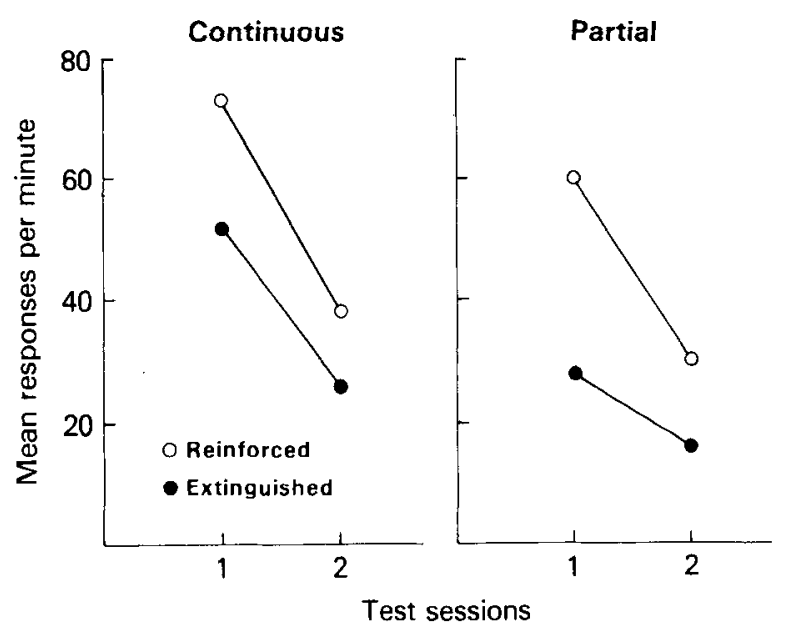

Figure 2. Experiment 2: Second-order responding after the reinforcer had been extinguished (solid symbols) or maintained (open symbols) following continuous (left panel) or partial reinforcement (right panel). compared with those that had been partially reinforced in the first test session $(T=29, p>.05)$.

Second, and more important, differential treatment of the S1s induced differential responding to the S2s with which they had been paired, regardless of the schedule of reinforcement. That effect was most clear in the first test session but was somewhat attenuated in the second test session because of extinction of responding to all stimuli. Statistical analysis over both sessions found significantly less responding to the $S 2$ whose $S 1$ had been extinguished rather than reinforced, after both continuous $(\mathrm{T}=17, \mathrm{p}=.05)$ and partial reinforcement $(\mathrm{T}=9, \mathrm{p}<$ $.01)$. Although the data suggest that partially reinforced S2s were more sensitive to the S1 treatments than were continuously reinforced $S 2 \mathrm{~s}$, statistical analysis found the effects to be of comparable size $(T=33, p>.05)$. These data provide no evidence that partial reinforcement increased the amount of S-R learning.

\section{EXPERIMENT 3}

This experiment explored a third condition which has been thought to reduce dependence on the current state of the reinforcer: degree of separation. Some authors have noted that instrumental behavior typically involves sequences of responses that differ in their distance from the goal. Consequently, responses early in the sequence may be primarily reinforced not by the ultimate goal, but rather by stimuli associated with later responses. Because there is evidence that changes in the goal do not have a complete effect on stimuli preceding the goal, some authors have suggested that behaviors reinforced by those stimuli (i.e., behaviors early in the chain) might be more immune to modifications in the value of the goal (Morgan, 1979; Rescorla, 1977).

This experiment explored the analogous possibility in Pavlovian conditioning. Pigeons were given a sequence of keylight S2s that terminated in a previously conditioned $\mathrm{S} 1$. Then that $\mathrm{S} 1$ was either extinguished or given additional reinforcement. Finally, responding was assessed to the various S2s. If distance from the reinforcer is an important variable, S2s earlier in the sequence should be less affected by changes in the value of the $S 1$.

\section{Method}

Subjects and Apparatus. The subjects were 16 female Carneaux pigeons maintained in individual cages at $75 \%$ of their free-feeding weights. They had previously participated in other autoshaping experiments, which had used chambers, response keys, and stimuli different from those employed here; their past treatments were arranged to be unrelated to those of the present experiment.

The apparatus consisted of four chambers identical in all important respects to those of the previous experiments. The stimuli were projected on the right-hand response key. These stimuli consisted of the illumination of quadrants of the key outlined by two orthogonal black lines, $1 \mathrm{~mm}$ thick, that formed an "X" on the key. Two colors, orange $(O)$ and magenta $(M)$, could be projected on the left- and right-hand quadrants. The other two quadrants could be illuminated by a plain white light $(\mathrm{W})$ or a white background containing a pattern of black dots (D), $.5 \mathrm{~mm}$ in diameter and spaced $1 \mathrm{~mm}$ apart. 
Procedure. Initially, all animals received first-order conditioning of W and D. On each of 3 days, there were 125 -sec reinforced presentations of each stimulus with a 2 -min ITI. On the following 2 days, 12 nonreinforced presentations each of $O$ and $M$ were added and the ITI was reduced to $1 \mathrm{~min}$.

On each of the next 3 days, the animals received a sequential second-order conditioning procedure, using either $\mathrm{D}$ or $\mathrm{W}$ as the reinforcer. For half the animals, each trial consisted of a 5-sec presentation of $\mathrm{O}$, followed by a 5-sec presentation of $\mathrm{M}$, followed by a 5-sec presentation of the reinforcer; for the other half, the positions of $\mathrm{O}$ and $\mathrm{M}$ were interchanged. Half of the animals in each subgroup received $\mathrm{D}$ as the reinforcer, and half received $\mathrm{W}$. Each session contained six such second-order trials, intermixed with two reinforced refresher trials each of $\mathrm{D}$ and $\mathrm{W}$, as well as six nonreinforced presentations of whichever pattern was not used as the reinforcer for $\mathrm{O}$ and $\mathrm{M}$. The mean ITI was $2 \mathrm{~min}$.

On each of the next 4 days, the animals received discriminative training on $\mathrm{D}$ and $\mathrm{W}$. Half of the animals in each of the above subgroups received 6 reinforced presentations of D and 24 nonreinforced presentations of $W$. The other half of the animals had the roles of $D$ and $W$ interchanged. The mean ITI was 1 min. Thus, this design ensured that the same S1 was used to train both the proximal and distal S2 within a subject. However, comparison of the effects of changes in the value of the $S 1$ on these $S 2 s$ was made between subjects; the $S 1$ for both the distal and proximal S2s was extinguished for half the animals but reinforced for the other half.

A single test session was given on the next day. The session began with 3 reinforced and 12 nonreinforced presentations of the appropriate pattern stimuli and continued with 6 nonreinforced test presentations of $O$ and $M$. The data of interest were the response rates to $\mathrm{O}$ and $\mathrm{M}$ as a function both of their proximity to the $\mathrm{SI}$ reinforcer and of the subsequent treatment of $\mathrm{S} 1$.

\section{Results}

First-order training of D and $\mathrm{W}$, as well as their initial discrimination from $\mathrm{O}$ and $\mathrm{M}$, proceeded smoothly. By the final day of that training, the mean response rates were 62.0 and 5.5 responses/min for the reinforced and nonreinforced stimuli, respectively. Second-order conditioning resulted in a substantial increase in responding to $O$ and M. However, two animals showed no signs of second-

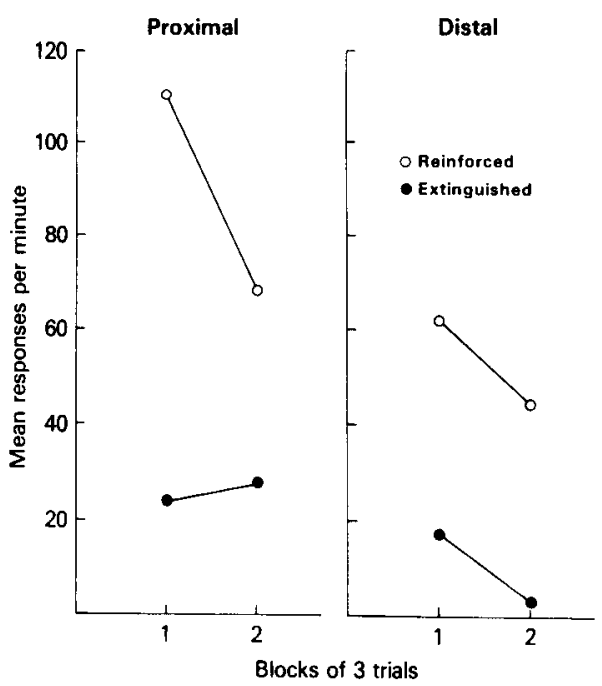

Figure 3. Experiment 3: Second-order responding after the reinforcer had been extinguished (solid symbols) or maintained (open symbols) as a function of the temporal interval between the tested stimulus and the reinforcer. order conditioning and consequently were dropped from the experiment. By the third day, the mean response rates for the remaining 14 animals were 46 and 90 , respectively, for the initial and second stimulus in the sequence $(T=4$, $\mathrm{p}<.01$ ). There were no reliable differences in responding as a function of the identity of the colors. Over the course of the 4 discrimination days, differential responding developed to the two pattern stimuli. On the final day of this phase, the mean response rates were 77.3 and 7.2 for the reinforced and nonreinforced stimulus, respectively.

Figure 3 shows the data of most interest, from the test presentations of the colors, following discrimination with the patterns. The left-hand panel shows those data for the color more proximal to the reinforcer during second-order conditioning; the right-hand panel shows the same data for the color more distal to that reinforcer. Responding to the color trained with a more proximal relation to the reinforcer remained higher than responding to the color trained with a more distal relation, whatever the subsequent treatment of the reinforcer (Ts $=0$, ps $<.02$ ). Of greater interest is the fact that extinction of a reinforcer produced a substantial reduction in responding for both the proximal $(U=2.5, p<.02)$ and the distal $(U=5.5$, $\mathrm{p}<.02$ ) stimulus.

These results lend no support to the proposition that a stimulus more removed from the reinforcer is less sensitive to changes in the value of that reinforcer.

\section{GENERAL DISCUSSION}

These experiments confirm the findings of other autoshaping experiments, that differential training of S1s produces differential responding to the $S 2 \mathrm{~s}$ with which they have been paired. Moreover, they found no evidence that this residual responding was enhanced by increasing the number of S2-S1 pairings, by using a schedule of partial reinforcement, or by increasing the temporal interval between $\mathrm{S} 2$ and $\mathrm{S} 1$.

An important feature of the present experiments is that they employed a design that ensured that the same reinforcer was used for conditioning stimuli that bore different relationships to it. Many earlier attempts to assess the impact of extended training or reinforcement schedules on the sensitivity of instrumental responding to reinforcer devaluation have used a between-subjects design. But interpretation of a between-subjects design is complicated by the fact that these variables may affect the susceptibility of the reinforcer to the operation used to change its value. For example, Adams (1982) found that the apparent insensitivity of leverpressing to a decrease in the reinforcer value following extended training could be explained by the fact that increased exposure to the reinforcer made the reinforcer more difficult to devalue. Similarly, Chen and Amsel (1980) found that partially reinforced responding was more resistant to reinforcer devaluation, but they also found the reinforcer to be less susceptible to devaluation following partial reinforcement. The use of the present design allows an assessment of the 
effect of the training parameters on the sensitivity of conditioned responding to reinforcer devaluation that is not confounded by differences in the ease with which the reinforcer is affected by postconditioning devaluation operations.

Despite the use of this design, the present studies provide no evidence that $S-R$ learning is amplified by any of the variables studied here. Of course, more extreme values of any of those variables might reveal an effect. For instance, a leaner partial reinforcement schedule, a greater number of stimuli intervening between $S 2$ and $S 1$, or a greater number of pairings of $\mathrm{S} 2$ with $\mathrm{S} 1$ might generate an S2 that is less sensitive to changes in S1.

Increasing the range of variation seems most attractive for the case of amount of training, because the values chosen in Experiment 1 did not, in fact, yield different levels of responding to the S2s. However, the use of second-order conditioning places important limits on the extent of training that can be carried out. It is well known that, with continued training, responding to $S 2$ declines because of the onset of conditioned inhibition. Both of the other variables studied had a demonstrable effect on conditioning, suggesting sensitivity of learning to the values chosen.

It should be noted that attaining different terminal levels of performance across different values of an independent variable has both positive and negative features. On the positive side, one can be confident of the animal's sensitivity to variation in the parameter under study. On the negative side, that sensitivity means that further manipulations (such as modifying the value of the reinforcer) must take place from baselines that are at different points on the response scale. It is always hazardous to interpret the magnitudes of changes induced in behaviors at different levels. Consequently, in experiments of the sort reported here, one can be most confident in drawing conclusions about whether or not responding continues to be sensitive to reinforcer devaluation, but must be more cautious in evaluating the magnitude of that sensitivity. But, for the range of variables studied here, that sensitivity persists, suggesting no qualitative change in the nature of second-order conditioning.

Consequently, the present results do not offer much assistance in understanding the insensitivity of instrumental performance to changes in the reinforcer. Elsewhere (Colwill \& Rescorla, 1985), we have offered alternative suggestions for explaining those data.

Finally, it should be noted that these data demonstrate a remarkable ability of the pigeon to form multiple associations with amazing rapidity. For instance, Experiment 1 found responding to $S 2$ to be sensitive to changes in its $\mathrm{S} 1$ after as few as six S2-S1 pairings in a context involving four S2 and two S1 events. Moreover, Experiment 2 found that intermixing six nonreinforced presentations of that same S2 did not prevent the learning. Apparently, pigeons are capable of simultaneously and rapidly forming quite a number of associations.

\section{REFERENCES}

Adams, C. D. (1982). Variations in the sensitivity of instrumental responding to reinforcer devaluation. Quarterly Joumal of Experimental Psychology, 34B, 77-98.

ADAMs, C. D., \& Dickinson, A. (1981a). Actions and habits: Variations in associative representations during instrumental learning. In N. E. Spear \& R. R. Miller (Eds.), Information processing in animals. Memory mechanisms. Hillsdale, NJ: Erlbaum.

ADAMs, C. D., \& Dickinśon, A. (1981b). Instrumental responding following reinforcer devaluation. Quarterly Journal of Experimental Psychology, 33B, 109-112.

CAPALDI, E. D., \& MYERS, D. E. (1978). Resistance to satiation of consummatory and instrumental performance. Leaming and Motivation, 9, 179-201.

ChEN, J. S., \& AMSEL, A. (1980). Recall (versus recognition) of taste and immunization against aversive taste anticipations based on illness. Science, 209, 851-853.

Colwill, R. M., \& Rescorla, R. A. (1985). Post-conditioning devaluation of a reinforcer affects instrumental responding. Journal of Experimental Psychology: Animal Behavior Processes, 11, 120-132.

GilhousEn, H. C. (1933). Fixation of excess distance patterns in the white rat. Journal of Comparative Psychology, 16, 1-24.

HaAs, R. B., Shessel, F. M., Willner, H. S., \& Rescorla, R. A. (1970). The effect of satiation following partial reinforcement. Psychonomic Science, 18, 296-297.

Holland, P. C., \& Rescorla, R. A. (1975). The effect of two ways of devaluing the unconditioned stimulus after first- and second-order appetitive conditioning. Journal of Experimental Psychology: Animal Behavior Processes, 1, 355-363.

IRWIN, F. W. (1971). Intentional behavior and motivation: A cognitive theory. Philadelphia: Lippincott.

Kimble, G. A., \& Perlmuter, L. C. (1970). The problem of volition. Psychological Review, 77, 361-384.

Morgan, M. J. (1974). Resistance to satiation. Animal Behaviour, 22, 449-466.

Morgan, M. J. (1979). Motivational processes. In A. Dickinson \& R. A. Boakes (Eds.), Mechanisms of learning and motivation. Hillsdale, NJ: Erlbaum.

NaIRNe, J. S., \& Rescorla, R. A. (1981). Second-order conditioning with diffuse auditory reinforcers in the pigeon. Learning and Motivation, 12, 65-91.

RASHOTTE, M. E. (1981). Second-order autoshaping: Contributions to the research and theory of Pavlovian reinforcement by conditioned stimuli. In C. M. Locurto, H. S. Terrace, \& J. Gibbon (Eds.), Autoshaping and conditioning theory. London: Academic Press.

Rashotte, M. E., Griffin, R. W., \& Sisk, C. L. (1977). Second-order conditioning of the pigeon's keypeck. Animal Learning \& Behavior, 5, 25-38.

ResCorla, R. A. (1977). Pavlovian second-order conditioning: Some implications for instrumental behavior. In H. Davis \& H. M. B. Hurwitz (Eds.), Operant-Pavlovian interactions. Hillsdale, NJ: Erlbaum.

Rescorla, R. A. (1979). Aspects of the reinforcer learned in secondorder Pavlovian conditioning. Journal of Experimental Psychology: Animal Behavior Processes, 5, 79-95.

Rescorla, R. A. (1980). Pavlovian second-order conditioning: Studies in associative learning. Hillsdale, $\mathrm{NJ}$ : Erlbaum.

Rizley, R. C., \& Rescorla, R. A. (1972). Associations in secondorder conditioning and sensory preconditioning. Journal of Comparative and Physiological Psychology, 81, 1-11.

Tolman, E. C. (1933). Sign-Gestalt or conditioned reflex? Psychological Review, 40, 246-255.

Tolman, E. C. (1948). Cognitive maps in rats and men. Psychological Review, 55, 189-208.

Wilson, C. L., Sherman, J. E., \& Holman, E. W. (1981). An aversion to the reinforcer differentially affects conditioned reinforcement and instrumental responding. Journal of Experimental Psychology: Animal Behavior Processes, 7, 165-174. 